Journal of Career Assessment

Volume 2/Number 1/Winter 1994/Pages 1-14

\title{
Unidimensionality Versus Multidimensionality of the Career Decision Scale: A Critique of Martin, Sabourin, Laplante, and Coallier
}

\author{
Kazuaki Shimizu \\ Kansai University \\ and \\ Fred W. Vondracek \\ The Pennsylvania State University \\ and \\ John Schulenberg \\ University of Michigan
}

\begin{abstract}
During the past 15 years the Career Decision Scale (CDS) has been used primarily to assess degree of indecision. Controversy has arisen around the issue of whether it should thus be considered a unidimensional measure or whether it is a multidimensional measure of several dimensions of career indecision. Although a number of studies reported significant empirical support for the multidimensionality of the CDS, a study by Martin, Sabourin, Laplante, and Coallier (1991) claimed to present evidence supporting its unidimensionality. The present study refutes those claims by demonstrating serious methodological and conceptual errors and omissions in the findings of Martin et al. (1991) and by presenting further empirical evidence in support of multidimensionality.
\end{abstract}

In his recent review entitled "Megatrends and Milestones in Vocational Behavior: A 20-Year Counseling Psychology Retrospective," Borgen (1991) reported that between 1975 and 1990, 34 articles on the topic of career indecision had been published in the Journal of Vocational Behavior alone. The main impetus for research on career indecision was undoubtedly the publication of the Career Decision Scale (CDS) by Osipow and his collaborators (Osipow, Carney, Winer, Yanico, \& Koschier, 1976b), and the publication of an article reporting the original exploratory factor analysis of the CDS (Osipow, Carney, \& Barak, 1976a). Other instruments have been introduced more recently to examine and measure different dimensions and antecedents of career indecision, including the Career Factors Inventory (Chartrand \& Robbins, 1990; Chartrand, Robbins, Morrill, \& Boggs, 1990), and the Career Decision Profile (Jones, 1989).

We would like to thank John R. Nesselroade and two anonymous editorial consultants for helpful comments on a draft of this manuscript. 
In spite of these various assessment efforts, Fitzgerald and Rounds' $(1989$, p.120) conclusion that "although there has been a general consensus that indecision is multidimensional, no agreement exists about the number and nature of these dimensions," is still valid. Indeed, the desirability of reliably identifying qualitatively different levels, dimensions, or antecedents of career indecision has been repeatedly confirmed, especially by researchers who are concerned about designing differential interventions (e.g., Savickas, 1989; Spokane, 1991). Use of the CDS as a measure of multiple dimensions of career indecision has continued to be controversial, however, in part because Osipow (1980) has discouraged the use of factor-based scales in view of findings from a number of studies (for a review, see Shimizu, Vondracek, Schulenberg, \& Hostetler, 1988) that were unable to produce factor solutions identical to the original factor analysis of the CDS (Osipow et al., 1976a). Nevertheless, Slaney was moved by this situation to complain that "...the tendency for researchers to use the CDS...[as a unidimensional measure] glosses over the issue of developing differential treatments based on the antecedents of career indecision" (1988, p. 63).

Although the dimensionality of the CDS has been the subject of a great deal of (often conflicting) research, Shimizu et al. (1988) found that the inconsistent findings of prior studies were largely due to widely differing methodological procedures used in the determination of the number of factors, in the estimates of communality, and in factor rotation procedures. They then demonstrated that, when rotational procedures were used that permitted oblique (i.e., correlated factors) solutions, the findings from six previous factor analytic studies of the CDS were considerably more similar to the original factor solution reported by Osipow et al. (1976a) than had been shown previously, mainly by using orthogonal solutions. Shimizu et al. (1988) then used exploratory factor analysis on a sample of 698 junior highschool and high-school students, again finding that a simple, four-factor solution was most appropriate. They named their factors Diffusion, Support, Approach-Approach, and External Barriers.

In an effort to further confirm this factorial structure of the CDS, Schulenberg, Shimizu, Vondracek, and Hostetler (1988), applied multiplegroup confirmatory factor analytic strategies to examine the fit of the previously reported four-factor model across four groups: Females and males, junior high-school and high-school students. Acceptable fit across the four groups was demonstrated, indicating that the factor structure was invariant with respect to grade-level and gender during adolescence. It should be noted here that the use of confirmatory factor analysis for this purpose is, contrary to Tinsley and Tinsley (1987), the most stringent test of the hypothesized factor structure. Based on these findings, Vondracek, Hostetler, Schulenberg, and Shimizu (1990) constructed four factor-based subscales of the CDS, nonoverlapping in items (linearly independent ${ }^{1}$ ), to

\footnotetext{
${ }^{1}$ The term "linearly independent" has been misinterpreted (e.g., Martin, Sabourin, Laplante, \& Coallier, 1991). It is derived from matrix algebra where, for example, vectors can be linearly independent but not orthogonal. The use of the term by Vondracek et al. (1990) referred not to the absence of linear correlation, but rather to the lack of overlap of items contributing to the four scales.
} 
be used in a longitudinal study of different dimensions of career indecision, providing additional support for the validity of this multidimensional use of the CDS.

Fitzgerald and Rounds (1989) suggested that the logical extension of these findings would be to examine them with new samples, including college students and adults. Using college students in a study of concurrent validity, Chartrand and Robbins (1990) compared CDS subscales constructed according to the findings of Osipow et al. (CDS-A; 1976a), Shimizu et al. (CDS-B; 1988), and the Career Factors Inventory (CFI; Chartrand \& Robbins, 1990). They concluded that the CDS-B subscales functioned better than the CDS-A subscales. Moreover, they reported that the three CDS-B subscales of Support, Approach-Approach, and External Barriers made unique, positive contributions to the prediction of career decidedness and career implementation. These findings lend further support to the CDS factorial structure reported by Shimizu et al. (1988).

\section{The Challenge of Martin, Sabourin, Laplante, and Coallier}

In spite of this converging evidence for the multidimensionality of the CDS, Martin, Sabourin, Laplante, and Coallier (1991) reported that their analyses led them to believe that a unidimensional model most adequately accounted for career indecision when measured by the CDS, and that use of the CDS total score should be promoted. They arrived at these conclusions after conducting a confirmatory factor analysis of a French version of the CDS administered to 589 college students. Specifically, their study was designed to examine the dimensionality of the 13 items of the CDS that were identified by Shimizu et al. (1988) as the "simple" model, and that constituted the items making up the recommended factor-based scales (Schulenberg et al., 1988; Vondracek et al., 1990).

Martin et al. (1991) examined three factor analytic models of the (13-item) CDS: The first model postulated was a unidimensional model, designed to test the assumption that the 13 items of the CDS are best represented by one factor. The second model was the four-factor model of Shimizu et al. (1988) included in order to confirm or disconfirm this model's applicability to the new sample. Finally, the third model postulated was a hierarchical model, designed to test the assumption that the 13 items represent 4 firstorder and 1 second-order factor. In their analyses they treated the CDS items as ordinal variables, used polychoric correlations to estimate correlations among CDS items, and used an asymptotic distribution free method (a modified generalized least-squares estimator proposed by Browne, 1984) to estimate the parameters of these models. In interpreting their findings, Martin et al. (1991) selected the unidimensional model as the model best representing their data.

Martin et al. (1991) made an important contribution to the growing literature on the dimensions of career indecision by specifically bringing attention to the issue of unidimensionality versus multidimensionality. Clearly, this is a critical issue in terms of career theory and practice; reasonable arguments can be made to support each side. Nevertheless, it is our contention that the conclusions reached by Martin et al. (1991) are 
flawed, and that, in answer to the question implied by the title of their article, they did not produce any "disconfirming evidence" for the fourfactor solution presented by Shimizu et al. (1988). In order to support our position, we shall discuss a number of issues that were raised, either explicitly or implicitly, by the Martin et al. (1991) paper, including (a) problems produced by the cross-cultural nature of their comparison; (b) methodological and conceptual errors in the rationale used for favoring the unidimensional model; and (c) other methodological omissions, inconsistencies, and flaws. Furthermore, we will present re-analyses of the Shimizu et al. (1988) data that replicate the analytic strategies of Martin et al. (1991), as well as additional analyses that bear upon the issue of the dimensionality of the CDS.

\section{Cross-Cultural Issues}

Although Martin et al. (1991) reported that the translation of the CDS into French was carried out quite carefully and diligently, we have some major concerns with their reported procedures. For example, after the English version was translated into French by two bilingual individuals, there apparently was no effort to "back-translate" the French version to consider the adequacy of the original translation. Most critically, however, they offered no evidence to support their assumption of equivalence of the English and French language versions of the CDS. Because they used research that utilized the French language CDS to question results obtained with the English language CDS, it is important to consider the adequacy of their cross-cultural methodology.

It is generally accepted that, in order to demonstrate that one is using essentially the same instrument in two different cultures, one must establish structural, and, if possible, metric equivalence between the two instruments (i.e., Poortinga, 1989). Thus, if the parameters in a factor analytic model, such as number of factors, salient versus non-salient factor loadings, factor variances, and unique variances are the same in both instruments, structural and metric equivalence is established. The appropriate method to examine such equivalence of two culturally different versions of an instrument is confirmatory factor analysis (CFA). For present purposes, it will suffice to note that both single group (e.g., Watkins, 1989) and multi-group CFAs (e.g., Joreskog, 1971) may be used in cross-cultural comparisons, depending on the specific purpose of the comparison.

Unfortunately, Martin et al. (1991) did not report any concern about either the structural or metric equivalence of their measure and the English language CDS used by Shimizu et al. (1988) and thus, they did not establish that they were using essentially the same measure. Consequently, it was literally impossible for them to offer any evidence disconfirming the four-factor model of Shimizu et al. (1988). What they could establish would be whether this four-factor model did or did not fit their (French CDS) data. Any comparative findings, however, would be difficult to interpret because they could be due, at least in part, to possible differences in the structural and/or metric properties of the French and English versions of the CDS and/or to any "real" cultural differences in career 
indecision between French-Canadians and Americans. In any case, the suggestion that these findings represent disconfirming evidence for the results of Shimizu et al. (1988) is clearly unwarranted.

\section{Multidimensional Versus Unidimensional Models: Methodological and Conceptual Issues}

There are a variety of methods that can be used to determine which of several alternative factor analytic models best fit a given factor model. Martin et al. (1991) used several fit indices, including the widely used ratio of the chi-square to its expected value $\left(\chi^{2} / d f\right)$, noting that a ratio of 2 to 1 or less is considered an acceptable fit (e.g., Bollen, 1989; Byrne, 1989; Joreskog \& Sorbom, 1988). Using this criterion with the 13-item CDS (i.e., Shimizu et al.'s, 1988, CDS) they estimated a unidimensional model and found the fit to be unsatisfactory $\left(\chi^{2} / d f=2.99\right)$. A second attempt, excluding item \#18, resulted in $\left(\chi^{2} / d f=1.99\right)$, just under the critical value of 2 . The multidimensional model (i.e., Shimizu et al.'s, 1988, model) produced a $\chi^{2} / d f$ of 1.73 , well below the critical value. Martin et al. (1991) were unable to estimate the fit of their hypothesized hierarchical model.

These findings, we believe, provided further evidence regarding the robustness of the four-factor model that we previously specified. That is, in light of the difficulties involved in replicating factor models in different samples (particularly a sample of considerably older respondents living in a different country), Martin et al.'s (1991) inability to reject our four-factor model in a confirmatory factor analytic test is evidence that can and will be added to the growing list of supportive findings regarding the four-factor model (see also, e.g., Chartrand \& Robbins, 1990). Therefore, it is more than a little surprising for Martin et al. (1991) to conclude that their unidimensional model should be preferred. Clearly, their rationale for their conclusion is contrary to the conventional wisdom in conducting such model comparisons, as well as otherwise unwarranted, as will be discussed below. Moreover, they urged continuing use of the CDS total score as an indicator of career indecision, in line with Osipow's $(1980,1987)$ recommendation. How they arrived at this conclusion is difficult to ascertain since they did not estimate a unidimensional model of the total (16-item) CDS, nor compared it with the 13-item version they did examine.

\section{Item \#18 is Excluded Because it Does Not "Fit"}

Questions must first be raised about the arbitrary exclusion of CDS item \# 18 on the basis that it was "problematic since it was involved in five of the nine SRs (standardized residuals) greater than 1.96" (Martin et al., 1991, p. 193). Item 18 refers to support regarding one's choice of college major, and is quite similar in content and structure to item 12 . On the face of it, item 18 seems innocent enough. By "problematic" it thus appears that Martin et al. (1991) mean "something that impedes an acceptable one-factor solution." Clearly, the large standardized residuals associated with item 18 indicate that the one-factor model provides a poor fit to the observed data, suggesting that additional factors are needed. The arbitrariness of this exclusion is further illustrated by the fact that reanalysis of the Shimizu et al. (1988) data (as discussed below) with item 18 excluded did not significantly 
improve the fit of the unidimensional model, but actually made the hierarchical model acceptable, while confirming the superiority of the multidimensional model. In any event, deleting an item because it refutes the desired model, and then reestimating the model with the same data, which unsurprisingly yields a more favorable verdict of the desired model, does not constitute acceptable model testing practices.

\section{The Multidimensional Model is Rejected Because "The Factor Intercorrelations Were Too Substantial"}

Martin et al.'s (1991) decision to favor their unidimensional model, in spite of the fact that the multidimensional model of Shimizu et al. (1988) provided a better fit to their data, apparently was based primarily on their interpretation of the meaning of the factor intercorrelations in the multidimensional model: "The factor intercorrelations were too substantial to conclude that the four-subscale model was an adequate representation of the observed data" (1991, p. 195). This is a curious, and again arbitrary, criterion to determine whether a model is an adequate representation of the data. If there were not four underlying, unobserved variables (factors), as the model specifies, Martin et al. (1991) would have been informed by the LISREL program (Joreskog \& Sorbom, 1988, 1989) they utilized that the estimation of the parameter matrices was not possible (i.e., not positivedefinite). But, because the four factors of the multidimensional model are linearly independent (though correlated), it was indeed possible to estimate the parameter matrix of factor correlations. The issue of factor intercorrelations is simply not an issue in model comparisons of the type conducted by Martin et al. (1991).

Indeed, when one is examining factorial invariance across different samples (or over time), it is essential to leave factor intercorrelations unrestricted precisely because it is likely that relations among factors will vary from sample to sample (e.g., see Meredith, 1964; Joreskog, 1971; Nesselroade, 1983). Martin et al.'s (1991) findings regarding the multivariate model represent a case in point (assuming that their findings came after demonstrating equivalence between the English and French versions of the CDS). They reported that in their confirmatory factor analysis of the multidimensional model the estimated correlations among factors ranged from .65 to .93 .

The factor intercorrelations from the exploratory factor analysis by Shimizu et al. (1988) ranged from .36 to .58. Using the same data in a confirmatory factor analysis Schulenberg et al. (1988) found the intercorrelations to range from .58 to .78 (because confirmatory factor analysis is more restrictive on the factor loading matrix, it typically yields higher factor correlations than does exploratory factor analysis). In addition, factor intercorrelations obtained by Chartrand and Robbins (1990) with a college student sample were found to range from .45 to .62.

The point here is not to dismiss Martin et al.'s (1991) concern with their factor intercorrelations that ranged from .65 to .93 . If we obtained their results, we would also be concerned, although in searching for the cause of these high correlations, we would have first considered the cross-cultural 
measurement issues that we discussed previously. Rather, the point is that variations in factor correlations across samples is to be expected, and thus, factor correlations alone bear little on confirmation or disconfirmation of a factor model (this is especially true when the model successfully converges on a solution and provides an excellent fit to the data, as was the case for Martin et al., 1991). Furthermore, factor intercorrelations vary according to the type of data inputted as well as the type of estimation procedures used. Indeed, different parameter estimation methods in LISREL yield different results even when the same data are used for such analyses (e.g., see Joreskog \& Sorbom, 1989, Table 7.4). Relatedly, although Martin et al. (1991) state that they also conducted the analyses using product-moment correlations and maximum likelihood estimation procedures, they did not report the factor intercorrelations based on these analyses-perhaps the correlations from these analyses were smaller.

It must also be noted that the size of the factor intercorrelations may be artificially elevated by the fact that several of the CDS items are complex and may thus be subject to multiple interpretations. This problem has been noted previously (e.g., Slaney, 1988; Vondracek et al., 1990) and Osipow has been urged to revise the CDS in order to produce both more and "cleaner" items in order to potentially increase the reliability of factor-based scales (Vondracek, 1991) and, perhaps, produce scales that are not as highly correlated as those obtained by Shimizu et al. (1988).

Finally, on this subject it is worth noting that disagreements exist about what constitutes "substantial" (cf. Martin et al., 1991, p. 195) factor intercorrelations. For example, Gorsuch (1988) maintained that "mathematically there is no criterion as to what level of correlation between two factors is 'best"' (p. 249). For some researchers correlations have to be above .70 to be considered substantial. The reason for this position becomes clear when it is realized that even with a correlation of .70, less than 50 percent of the variance in the two variates is shared. Thus, two factors that are correlated at .70 cannot, under any circumstances, be assumed to be measuring an identical underlying construct, thus warranting their separate measurement.

\section{When 16 Equals 13 (or is it 12?)}

After accepting their modified unidimensional model over the multidimensional model, Martin et al. (1991) urged that "the use of the CDS total score should be promoted" (p. 195). Consistent with Osipow's (1980, 1987) recommendation, the only reasonable assumption is that Martin et al. (1991) mean total score to indicate a score based on all 16 CDS items. This is another curious assertion, for they did not test a unidimensional model with all 16 items, and the one they tested with 13 items provided an inferior and unacceptable fit to the data. It appears that their rationale for promoting the use of the CDS total score based on 16 items is that an acceptable unidimensional model (that was not superior to the multidimensional model) was obtained with 12 CDS items, only after the "problematic" item 18 was removed. Of course, the only possible alternative, albeit unstated in the Martin et al. (1991) article, is that they are recommending the use of a 
modified CDS total score based only on 12 items arbitrarily selected from the 16 items. Now that would really be a curious assertion.

\section{The Heart of the Matter-Or Why it Now Matters Little What Osipow Originally Intended}

In constructing the CDS, what did Osipow and his colleagues have in mind-a unidimensional or multidimensional construct of career indecision? According to Martin et al. (1991): "Despite allegations to the contrary (Vondracek et al., 1990, p. 98), no attempts were made to construct a fourfactor scale from the start" (p. 196). This statement is unnecessarily strident, and of course, we made no allegations about initial attempts to construct a four-factor scale. What we did say was that "the CDS was specifically designed to examine different dimensions of career indecision" (Vondracek et al., 1990, p. 98), a reasonable interpretation based on the first article regarding the CDS (Osipow et al., 1976). Indeed, one of the original authors of the CDS (Winer, 1992) reported that "The CDS is an instrument that was intended to describe types of career indecision to be used as the basis for an intervention system, but that has been used primarily to assess degree of indecision rather than type" (p. 373).

Nevertheless, with all due respect to Professor Osipow and his colleagues, it matters little what they originally intended when constructing the CDS. Rather, what matters most now is that the relevant literature is becoming filled with evidence from several empirical studies (Martin et al., 1991, included) to indicate that the CDS reliably measures four dimensions of career indecision, and that there is preliminary empirical evidence (offered by Vondracek et al., 1990) indicating that the distinct dimensions "may bear important developmental and clinical implications" (Martin et al., 1991, p. 195). Given a choice, one would ordinarily prefer dimensions to be theoretically-derived rather than empirically-derived post-hoc. But, to prejudge the former as inherently superior to the latter is to fall prey to the false dichotomy between theoretical and empirical pursuits (e.g., see Cattell's [1966] discussion regarding the "inductive-hypothetico-deductive spiral"). Regardless of how the dimensions were derived, and regardless of the other measures of career indecision that have been and will be developed, the heart of the matter is this: empirical evidence consistently indicates the existence and importance of the four correlated yet distinct CDS dimensions (Diffusion, Support, Approach-Approach, and External Barriers).

\section{Other Methodological Omissions, Inconsistencies, and Flaws}

In addition to the fatal flaws regarding Martin et al.'s (1991) failure to first establish equivalence between the French and English versions and their unscientific rationale for choosing the unidimensional model, we found other crucial methodological omissions, inconsistencies, and flaws in the Martin et al. (1991) article. In particular, one obvious question that was omitted is simply: how many factors were indicated by the data? That is, what is the verdict based on such indices as the number of eigenvalues greater than one, the scree test, and the Tucker-Lewis reliability coefficient? Do these indices converge to suggest only one factor? Based on the data we have seen, our guess is that the answer to the last question is negative. 
Several other omissions further compromised the presentation of Martin et al. (1991) For example, the actual correlations among the items would have been helpful. Also, because they make a point of their data being nonnormal, it would have been very useful to see skewness and kurtosis estimates to verify their point. This is especially important, given that their nonnormality assumptions could have significantly affected their findings. Furthermore, many crucial statistical values (e.g., chi-square values and degrees of freedom for the first unidimensional model and for the multidimensional model, RMR values for the multidimensional model) were omitted, making it difficult to judge, on our own, the relative adequacy of the various models. Among the other salient concerns regarding the findings and conclusions of Martin et al. (1991), we will mention briefly only two that are particularly significant: (a) treatment of the CDS items as ordinal variables, and (b) interpretation of failed attempts to estimate the hierarchical model.

CDS items are ordinal variables-yes and no? Martin et al. (1991) indicated that because responses to the CDS items are arranged according to a Likert-type scale, the items should be treated as ordinal rather than interval, thus making it necessary to use polychoric correlations rather than product-moment correlations as input into the LISREL program. In our judgment, the CDS items are not ratio variables, but it is not clear whether they represent interval variables. What is clear, however, is that if indeed the CDS items are only ordinal variables, then it would be highly inappropriate to construct a total CDS score by simply summing the responses - that is, to derive a total score by simply summing the responses, it is necessary to postulate that the items are interval variables, otherwise differences among total scores would not be meaningful. Thus, by promoting the use of a summated CDS total score, it appears that Martin et al. (1991) are in the unenviable position of advocating, in the same paper, that the CDS items are and are not ordinal variables.

When you hear hoofs beating the ground, first look for horses, not zebras. Martin et al. (1991) were unable to get the LISREL program to estimate their hierarchical model (in which the 4 factors from the multidimensional model form a single higher-order factor). According to their description, they encountered Heywood cases (i.e., instances of negative residual variance) and instances of underidentification (although the model is algebraically overidentified). We agree that this is a puzzle, but based on our years of experience with the sometimes "temperamental" LISREL program, our first thought as to why the lack of convergence occurred would have been to look for obvious explanations. For example, perhaps the model simply provided a very bad fit to the data. Perhaps there exists more than one higher-order factor (the factor intercorrelations suggest as much). In contrast, Martin et al. (1991) apparently bypassed the more obvious explanations and instead pursued a far more speculative explanationthat is, that the correlations among the first-order factors were too high, which contributed to difficulties in estimating unique factor variances, which in turn contributed to the illusion of underidentification of various parameters. They then take this speculative explanation to provide further 
support that there is "too much correlation among the first-order factors" (p. 195), a familiar assertion. But let's not lose the point: regardless of why the hierarchical model did not converge, its lack of convergence has nothing to do with the multidimensional model, which did converge and did provide an excellent fit to the data.

\section{Reanalysis of the Data of Shimizu et al. (1988)}

Even when allowance is made for legitimate differences in interpreting the meaning of "substantial" factor intercorrelations, and when the problems of cross-cultural comparison are ignored, it is difficult to find any evidence in the study by Martin et al. (1991) that would qualify as "disconfirming" the multidimensionality of the CDS, in general, or the factor structure reported by Shimizu et al. (1988). However, it could legitimately be asked: What would happen if the Martin et al.'s (1991) analyses were applied to the Shimizu et al.'s (1988) data?

Before presenting our re-analyses it should be noted that there are some important features related to the nonnormality assumption made by Martin et al. (1991) that could significantly affect the results of their factor analysis, such as item skewness and kurtosis, but were not reported by them. In Table 1 we present the relevant item statistics for the 13 CDS items retained in the simple model of Shimizu et al. (1988), including their skewness and kurtosis. Note that there is little indication of extreme nonnormality.

In accordance with the Martin et al. (1991) study, three factor analytic models were examined via confirmatory factor analysis of polychoric correlations, using LISREL 7 (Joreskog \& Sorbom, 1989). The unidimensional model postulated by Martin et al. (1991) allowed all 13 items of the CDS retained by Shimizu et al. (1988) to load on one unique factor; the multidimensional model used was that identified by Shimizu et al. (1988), and the hierarchical model postulated by Martin et al. (1991) provided for the existence of a second-order factor that would account for the correlations observed among the 4 first-order factors. The models were estimated using the generally weighted least squares (WLS) method. In order to precisely replicate the analyses of Martin et al. (1991), all analyses were then repeated, but only the 12 items retained by them were used (i.e., item 18 was deleted). The findings for the three models, using 13 items and 12 items, respectively, are presented in Table 2.

The fit of each model was evaluated through use of the usual indices: the goodness-of-fit index (GFI); the adjusted goodness of fit index (AGFI); the root mean square residual (RMSR); the $\chi^{2}$; the ratio of the $\chi^{2}$ to its expected value $\left(\chi^{2 / d f}\right)$; and the Bentler-Bonett nonnormed fit index (BB). As can be seen in the table, both the multidimensional and hierarchical models had acceptable fit on most indices, with the multidimensional model having a slightly better fit, especially on the ratio of the chi-square to its expected value. There, only the multidimensional model achieved a value below the critical value of 2.00 (when 13 items were used); when item 18 was deleted, the multidimensional and hierarchical models had acceptable values. The unidimensional model was rejected as having the worst fit of any of the models. Factor intercorrelations for the multidimensional model ranged 
Table 1

Item Statistics of the CDS (13 Items of Simple Model; $N=703^{*}$ )

\begin{tabular}{|c|c|c|c|c|c|c|c|c|}
\hline \multirow[b]{2}{*}{ Variable } & \multirow{3}{*}{$\frac{M}{2.13}$} & \multirow{3}{*}{$\begin{array}{c}\boldsymbol{S D} \\
1.02\end{array}$} & \multirow{3}{*}{$\frac{\text { Skewness }}{0.44}$} & \multirow{3}{*}{$\frac{\text { Kurtosis }}{-0.98}$} & \multicolumn{4}{|c|}{ Category frequency } \\
\hline & & & & & $\mathbf{1}$ & 2 & 3 & 4 \\
\hline CDS 3 & & & & & 243 & 215 & 159 & 86 \\
\hline $\operatorname{CDS} 4$ & 2.44 & 1.12 & 0.06 & -1.35 & 189 & 176 & 175 & 163 \\
\hline CDS 5 & 1.65 & 0.93 & 1.27 & 0.49 & 421 & 156 & 76 & 50 \\
\hline CDS 6 & 1.79 & 1.06 & 1.02 & -0.40 & 406 & 123 & 92 & 82 \\
\hline CDS 7 & 1.88 & 1.03 & 0.80 & -0.66 & 349 & 161 & 123 & 70 \\
\hline CDS 8 & 1.98 & 1.02 & 0.66 & -0.77 & 297 & 201 & 130 & 75 \\
\hline $\operatorname{CDS} 9$ & 1.85 & 0.98 & 0.90 & -0.32 & 335 & 205 & 98 & 65 \\
\hline CDS 11 & 1.98 & 1.07 & 0.68 & -0.87 & 316 & 175 & 122 & 90 \\
\hline CDS 12 & 2.12 & 1.06 & 0.48 & -1.04 & 260 & 200 & 145 & 98 \\
\hline CDS 15 & 2.31 & 1.06 & 0.24 & -1.16 & 198 & 214 & 169 & 122 \\
\hline $\operatorname{CDS} 16$ & 2.14 & 1.05 & 0.41 & -1.09 & 252 & 193 & 163 & 95 \\
\hline CDS 17 & 2.25 & 1.06 & 0.28 & -1.16 & 218 & 200 & 178 & 107 \\
\hline CDS 18 & 2.27 & 1.05 & 0.24 & -1.16 & 211 & 200 & 185 & 107 \\
\hline
\end{tabular}

*The original factor analysis by Shimizu et al. (1988) reported an $N$ of 698 . The difference is due to the recovery of some lost data. Our analyses confirmed that the sample of 703 is virtually identical to the original sample of 698 .

Table 2

Goodness-of-Fit Results for 3 Models Using Polichoric Correlations

\begin{tabular}{lccccccc}
\hline Model & GFI & AGFI & RMSR & BBI & $\chi^{\mathbf{2}}$ & $\boldsymbol{d f}$ & $\chi^{\mathbf{2} / \boldsymbol{d} \boldsymbol{f}}$ \\
\hline Using 13 items & & & & & & & \\
$\quad$ Unidimensional & .96 & .95 & .07 & .92 & 301.16 & 65 & 4.63 \\
Multidimensional & .99 & .98 & .04 & .97 & 110.66 & 59 & 1.88 \\
$\quad$ Hierarchical & .98 & .98 & .05 & .97 & 129.76 & 61 & 2.13 \\
Using 12 items & & & & & & & \\
$\quad$ Unidimensional & .97 & .96 & .07 & .94 & 213.56 & 54 & 3.96 \\
$\quad$ Multidimensional & .99 & .98 & .04 & .97 & 87.98 & 48 & 1.83 \\
$\quad$ Hierarchical & .99 & .98 & .04 & .97 & $\mathbf{9 4 . 3 7}$ & 50 & 1.89 \\
\hline
\end{tabular}


from .64 to .83 (13 items), and .61 to .83 (12 items). For the hierarchical model (first-order factors) the corresponding values ranged from .63 to .80 (13 items), and .58 to .82 (12 items).

\section{Conclusion}

The CDS has been popular, most likely, because it has filled a need. At the same time, it has stimulated intellectual debate and research regarding the dimensions of career indecision. Although questions about the dimensionality of the CDS have been raised from time to time, evidence has been accumulating that suggests that its use as a unidimensional indicator of career indecision is questionable on a number of conceptual, psychometric, and methodological grounds. Moreover, evidence produced by the present authors, as well as by others, has confirmed that multiple dimensions of career indecision may be measured by the CDS. Concerns remain, however, that factor analytically derived subscales of the CDS may have low reliability because of the small number of items per scale (Vondracek, 1991). In addition, confirmatory factor analyses with new and different samples will be needed to extend the generalizability of previous findings.

Further empirical research is required to ascertain whether the underlying dimensions of the CDS are subject to developmental change and whether they are invariant across gender. Preliminary evidence suggests that the dimensions are continuous between adolescence and young adulthood (Chartrand \& Robbins, 1990; Schulenberg et al., 1988) and across gender (Schulenberg et al., 1988). Whether these dimensions are continuous across cultures is an open question, although the findings of Martin et al. (1991) (albeit only preliminary because they did not establish equivalence between the English and French versions of the CDS) suggest some cross-cultural continuity as does the study of a South African sample by Watson, Foxcroft, and Stead (1991). Clearly, however, the findings of Martin et al. (1991) do not constitute acceptable evidence for the unidimensionality of the CDS, for the reasons discussed earlier. At this point in time, after literally dozens of studies have been conducted with the CDS, and notwithstanding the claims of Martin et al. (1991), there is sound evidence that the CDS can form the basis for reliably identifying dimensions of career indecision. We can only hope that Osipow will be motivated sooner, rather than later, to consider "further expansion of the items for each factor" (Osipow, 1991, p. 332). Otherwise, new instruments like the Career Factors Inventory of Chartrand, Robbins, Morrill, and Boggs (1991) may replace the CDS.

\section{References}

Bollen, K. A. (1989). Structural equations with latent variables. New York: Wiley.

Borgen, F. H. (1991). Megatrends and milestones in vocational behavior: A 20-year counseling psychology retrospective. Journal of Vocational Behavior, 39, 263-290.

Browne, M. W. (1984). Asymptotically distribution-free methods for the analysis of covariance structures. British Journal of Mathematical and Statistical Psychology, 37, 62-83.

Byrne, B. M. (1989). A primer of LISREL: Basic applications and programming for confirmatory factor analytic models. New York: Springer Verlag.

Cattell, R. B. (1966). Psychological theory and scientific method. In R. B. Cattell (Ed.), Handbook of multivariate experimental psychology (pp. 1-18). Chicago: Rand McNally. 
Chartrand, J. M., \& Robbins, S. B. (1990). Using multidimensional career decision instruments to assess career decidedness and implementation. Career Development Quarterly, 39(2), 166-177.

Chartrand, J. M., Robbins, S. B., Morrill, W. H., \& Boggs, K. (1990). Development and validation of the Career Factors Inventory. Journal of Counseling Psychology, 37, 491-501.

Fitzgerald, L. F., \& Rounds, J. B. (1989). Vocational behavior, 1988: A critical analysis. Journal of Vocational Behavior, 35, 105-163.

Gorsuch, R. L. (1988). Exploratory factor analysis. In J. R. Nesselroade \& R. B. Cattell (Eds.), Handbook of multivariate experimental psychology (2nd ed.) (pp. 231-258). New York: Plenum.

Jones, L. K. (1989). Measuring a three-dimensional construct of career indecision among college students: A revision of the Vocational Decision Scale-The Career Decision Profile. Journal of Counseling Psychology, 36, 477-586.

Joreskog, K. G. (1971). Simultaneous factor analysis in several populations. Psychometrika, $36,409-426$.

Joreskog, K. G., \& Sorbom, M. (1988). PRELIS: A program for multivariate data screening and data summarization. A preprocessor for LISREL (2nd ed.). Mooresville, IN: Scientific Software, Inc.

Joreskog, K. G., \& Sorbom, M. (1989). LISREL 7: User's reference guide. Mooresville, IN: Scientific Software, Inc.

Martin, F., Sabourin, S., Laplante, B., \& Coallier, J. C. (1991). Diffusion, Support, Approach, and External Barriers as distinct theoretical dimensions of the Career Decision Scale: Disconfirming evidence? Journal of Vocational Behavior, 38, 187-197.

Meredith, W. (1964). Notes on factorial invariance. Psychometrika, 29, 177-185.

Nesselroade, J. R. (1983). Temporal selection and factor invariance in the study of development and change. In P. B. Baltes \& O.G. Brim, Jr. (Eds.), Lifespan development and behavior (Vol. 5, pp. 59-87). New York: Academic Press.

Osipow, S. H. (1980). Manual for the Career Decision Scale. Columbus, OH: Marathon Consulting and Press.

Osipow, S. H. (1987). Manual for the Career Decision Scale (rev. ed.). Odessa, FL: Psychological Assessment Resources.

Osipow, S. H. (1991). Response to Vondracek, Dorn, and Hackett. Journal of Counseling and Development, 70, 332-333.

Osipow, S. H., Carney, C. G., \& Barak, A. (1976a). A scale of educational-vocational undecidedness: A typological approach. Journal of Vocational Behavior, 9, 233-243.

Osipow, S. H., Carney, C. G., Winer, J. L., Yanico, B. J., \& Koschier, M. (1976b). The Career Decision Scale (3rd rev. ed.). Columbus, OH: Marathon Consulting and Press.

Poortinga, Y. H. (1989). Equivalence of cross-cultural data: An overview of basic issues. International Journal of Psychology, 24, 737-756.

Savickas, M. L. (1989). Annual review: Practice and research in career counseling and development, 1988. Career Development Quarterly, 38, 100-134.

Schulenberg, J. E., Shimizu, K., Vondracek, F. W., \& Hostetler, M. (1988). Factorial invariance of career indecision dimensions across junior high and high-school males and females. Journal of Vocational Behavior, 33, 63-81.

Shimizu, K., Vondracek, F. W., Schulenberg, J. E., \& Hostetler, M. (1988). The factor structure of the Career Decision Scale: Similarities across selected studies. Journal of Vocational Behavior, 32, 213-225.

Slaney, R. B. (1988). The assessment of career decision making. In W. B. Walsh \& S. H. Osipow (Eds.), Career Decision Making (pp. 33-76). Hillsdale, NJ: Erlbaum.

Spokane, A. R. (1991). Career intervention. Englewood Cliffs, NJ: Prentice Hall.

Tinsley, H. E. A., \& Tinsley, D. J. (1987). Uses of factor analysis in counseling psychology research. Journal of Counseling Psychology, 34(4), 414-424.

Vondracek, F. W. (1991). Osipow on the Career Decision Scale: Some comments. Journal of Counseling and Development, 70, 327. 
Vondracek, F. W., Hostetler, M., Schulenberg, J. E., \& Shimizu, K. (1990). Dimensions of career indecision. Journal of Counseling Psychology, 37(1), 98-106.

Watson, M. B., Foxcroft, C. D., \& Stead, G. B. (1991). Factor analysis of the Career Decision Scale on South African high-school students. Psychological Reports, 69, 1083-1088.

Watkins, D. (1989). The role of confirmatory factor analysis in cross-cultural research. International Journal of Psychology, 24, 685-701.

Winer, J. L. (1992). The early history of the Career Decision Scale. Career Development Quarterly, 40, 369-375. 